\title{
Insertion depth of oral stomach tubes may affect the fermentation parameters of ruminal fluid collected in dairy cows ${ }^{1}$
}

\author{
J. S. Shen, Z. Chai, L. J. Song, J. X. Liu, ${ }^{2}$ and Y. M. Wu \\ Institute of Dairy Science, MoE Key Laboratory of Molecular Animal Nutrition, College of Animal Science, Zhejiang University, Hangzhou 310058, \\ P. R. China
}

\section{ABSTRACT}

Six rumen-fistulated dairy cows were used in 2 trials to validate the technique for the collection of ruminal fluid by an oral stomach tube (OST). Trial 1 was conducted to compare the differences of ruminal fermentation parameters among rumen sites (cranial dorsal, cranial ventral, central, ventral, caudal dorsal, and caudal ventral). The ruminal fluid was collected once per day for 3 consecutive days through rumen cannula $(\mathrm{RC})$. The samples were analyzed for $\mathrm{pH}$, volatile fatty acids (VFA), ammonia $\mathrm{N}$, sodium, potassium, calcium, chloride, and phosphorus concentrations. The ruminal fermentation parameters varied significantly among rumen sites. Compared with the central or ventral rumen, the cranial dorsal rumen had significantly higher $\mathrm{pH}$, ammonia, and sodium concentrations and lower acetate, propionate, and butyrate concentrations, indicating that the sampling site may be one of the main factors contributing to the difference in ruminal fermentation parameters between the samples collected via the OST and RC. In trial 2, the fermentation parameters of ruminal fluid collected via OST at 2 insertion depths (180 or $200 \mathrm{~cm}$ ) were compared with those of ruminal fluid collected via RC (ventral sac). Ruminal fluid was collected once per week at 5 to 6 $\mathrm{h}$ after morning feeding. When the OST was inserted to a depth of $180 \mathrm{~cm}$, the OST head was located in the cranial dorsal (atrium) of the rumen. The ruminal fluid collected via the OST had higher $\mathrm{pH}$ and sodium concentrations but lower VFA, potassium, calcium, and phosphorus concentrations than that collected via RC. However, when the OST was inserted to a depth of 200 $\mathrm{cm}$, the OST head could pass through the front rumen pillar and reach the central rumen for sampling. No differences were found in $\mathrm{pH}, \mathrm{VFA}$, ammonia $\mathrm{N}$, and

\footnotetext{
Received March 3, 2012.

Accepted June 22, 2012.

${ }^{1}$ This work was supported by grants from the National Basic Research Program of China, Ministry of Science and Technology, P. R. China (Beijing, China; grant no. 2011CB100801) and China Agriculture Research System (CARS-37).

${ }^{2}$ Corresponding author: liujx@zju.edu.cn
}

ion concentrations between the samples collected via the 2 sampling methods. These results indicated that the OST should be inserted to reach the central rumen to obtain representative rumen fluid samples.

Key words: insertion depth, oral stomach tube, fermentation parameter, dairy cow

\section{INTRODUCTION}

The collection of ruminal fluid is important in disease diagnosis, therapy, and scientific research in ruminants (Geishauser, 1993; Geishauser and Gitzel, 1996; Hofírek and Haas, 2001). Rumen cannulation (RC) is the preferred method for obtaining representative samples of ruminal fluid, but its use is limited in scientific research (Nocek, 1997). Rumenocentesis (rumen puncture) and the use of an oral stomach tube (OST) are 2 techniques that can be used in field conditions (Duffield et al., 2004). Compared with rumenocentesis, OST is simpler, quicker and less invasive (Geishauser, 1993; Duffield et al., 2004) and has been used to collect ruminal fluid by scientific researchers (Einarson et al., 2004; Hook et al., 2009; Lodge-Ivey et al., 2009).

Opinions relative to the validity and representativeness of ruminal fluid collected via OST are controversial. Some authors (Nordlund and Garrett, 1994; Duffield et al., 2004) believe that the OST technique is susceptible to saliva contamination, resulting in higher ruminal fluid $\mathrm{pH}$ than samples collected from RC. Other authors (Geishauser, 1993; Geishauser and Gitzel, 1996; Lodge-Ivey et al., 2009) believe that saliva contamination is rarely a factor, and ruminal fermentation parameters are similar for samples collected via OST and RC. This controversy may be attributable to the different insertion depths of the OST. Bryant and Robinson (1968), Duffield et al. (2004), and Li et al. (2009) demonstrated that the cranial dorsal (atrium) of the rumen usually has a higher $\mathrm{pH}$ compared with other sites, which is considered the result of rumination and the consequent entry of increased volumes of saliva into the rumen (Bryant, 1964). Additionally, the flow of saliva induced by probing passes from the reticulum to the atrium of the rumen, also contributing to the 
higher $\mathrm{pH}$ of the cranial dorsal rumen (Dirksen, 1975). Therefore, we speculated that the insertion depth of the OST may influence the fermentation parameters of the ruminal fluid collected in dairy cows.

The objectives of this study were to compare the ruminal fermentation parameters among the rumen sites and to investigate the fermentation parameters of ruminal fluid collected by OST under 2 different insertion depths in comparison with those of the ruminal fluid collected via RC.

\section{MATERIALS AND METHODS}

\section{Animals and Diets}

The experimental procedures used in this study were approved by the Animal Care Committee, Zhejiang University (Hangzhou, China), and were in accordance with the university's guidelines for animal research. The health of the cows was monitored continuously before and during the experimental period.

Six Chinese Holstein cows [BW $=604 \pm 36 \mathrm{~kg}$; milk yield $=15 \pm 2.4 \mathrm{~kg}$ (mean $\pm \mathrm{SD}$ ) at the beginning of the study] fistulated with $\mathrm{RC}$ were housed in individual tie-stalls fitted with rubber mats and had continuous access to water from water bowls. The ingredient (DM basis) of the diets contained $17 \%$ corn silage, $10 \%$ Chinese wild rye, $28 \%$ alfalfa hay, $27 \%$ corn grain, $5 \%$ wheat bran, $5 \%$ soybean meal, $4 \%$ cottonseed meal, $0.25 \%$ calcium carbonate, $1.25 \%$ calcium phosphate, $1.0 \%$ sodium bicarbonate, $0.5 \%$ salt, and $1.0 \%$ premix (vitamins and microelements) and were offered as TMR for approximately $5 \%$ feed refusal in equal amounts at 0700 and $1900 \mathrm{~h}$. The TMR contained $14.3 \% \mathrm{CP}, 38.6 \%$ $\mathrm{NDF}$, and $24.1 \% \mathrm{ADF}$ with $\mathrm{NE}_{\mathrm{L}}$ of $1.56 \mathrm{Mcal} / \mathrm{kg}$ of DM. The cows were milked twice daily at 0730 and $1930 \mathrm{~h}$, and the individual milk yield was recorded for each milking.

\section{Experimental Design and Sampling Methods}

The study lasted for $9 \mathrm{wk}$ and included 2 trials. The OST (Figure 1A) used in this study was designed and manufactured by Anscitech Co. Ltd. (Wuhan, China), a modified type of that used by Geishauser (1993). The main parameters were as follows: length of probe head $=15 \mathrm{~cm}$, mass of probe head $=435 \mathrm{~g}$, diameter of probe head $=3.1 \mathrm{~cm}$, number of holes in the probe head $=$ 160 , diameter of the holes $=2.5 \mathrm{~mm}$, and diameter of spiral spring $=18 \mathrm{~mm}$.

Trial 1. During wk 1, ruminal fluid samples were collected through RC once per day for 3 consecutive days from 6 sites of the rumen, namely, the cranial dor- sal, cranial ventral, central, ventral, caudal dorsal, and caudal ventral (Figure 1C) rumen at approximately 5 to $6 \mathrm{~h}$ after morning feeding. Sample collection was performed using a probe (Figure 1B; Anscitech Co. Ltd.) as described previously by Duffield et al. (2004), with some modifications. To reduce performance error among the cows, all samples were collected by 1 person, and every sampling site was controlled accurately. The same procedure was followed at each collection. The cranial dorsal sample was obtained by advancing the probe through the fistula toward the head (insertion depth $=55 \mathrm{~cm}$ ). The cranial ventral sample was collected by inserting the probe at a $60^{\circ}$ angle toward the front feet through the fistula (insertion depth $=$ $55 \mathrm{~cm}$ ). The central rumen was sampled by advancing the probe almost horizontally through the fistula site at a $90^{\circ}$ angle to the fistula and no further than the midline of the cow (insertion depth $=25 \mathrm{~cm}$ ). The ventral rumen was sampled by advancing the probe almost vertically through the fistula site at a $90^{\circ}$ angle to the fistula (insertion depth $=45 \mathrm{~cm}$ ). The caudal dorsal sample was obtained by advancing the probe through the fistula toward the hips (insertion depth $=30 \mathrm{~cm}$ ). The caudal ventral rumen was sampled by inserting the probe at a $60^{\circ}$ angle toward the back feet through the fistula (insertion depth $=50 \mathrm{~cm}$ ). When ruminal fluid samples were collected from each site, the first $150 \mathrm{~mL}$ was discharged into a $1,000-\mathrm{mL}$ plastic beaker to avoid ruminal fluid contamination from the prior site. After sampling each cow, the ruminal fluid in the plastic beaker was turned back into the rumen and the OST was washed using running water.

Trial 2. In wk 2, the insertion depths of the OST from the incisors to different rumen sites (cardia of rumen, front rumen pillar, central rumen, and distal point of caudal-dorsal rumen) were measured by inserting the OST and palpation via the fistula before evening feeding. According to the measuring results, the OST was inserted to a depth of 180 or $200 \mathrm{~cm}$ for ruminal fluid collection at 5 to $6 \mathrm{~h}$ after morning feeding once per week. The ruminal fluid was collected through spontaneous efflux after aspirating with a modified $60-\mathrm{mL}$ syringe (Figure 1A). The OST was withdrawn at a length of 30 to $100 \mathrm{~cm}$ and reintroduced to its original depth when no ruminal fluid was siphoned off as described by Geishauser (1993). From wk 3 to 6 , the OST was inserted to a depth of $180 \mathrm{~cm}$. Samples were taken as 4 batches of $150 \mathrm{~mL}$ (the first, second, third, and fourth batches) for comparison. From wk 7 to 9 , the OST was inserted to a depth of $200 \mathrm{~cm}$. Depending on the results of the samples taken at a depth of $180 \mathrm{~cm}$, the first 150 $\mathrm{mL}$ of ruminal fluid was discarded and then $150 \mathrm{~mL}$ of ruminal fluid was collected. After sampling via OST, the rumen ventral fluid was collected immediately 

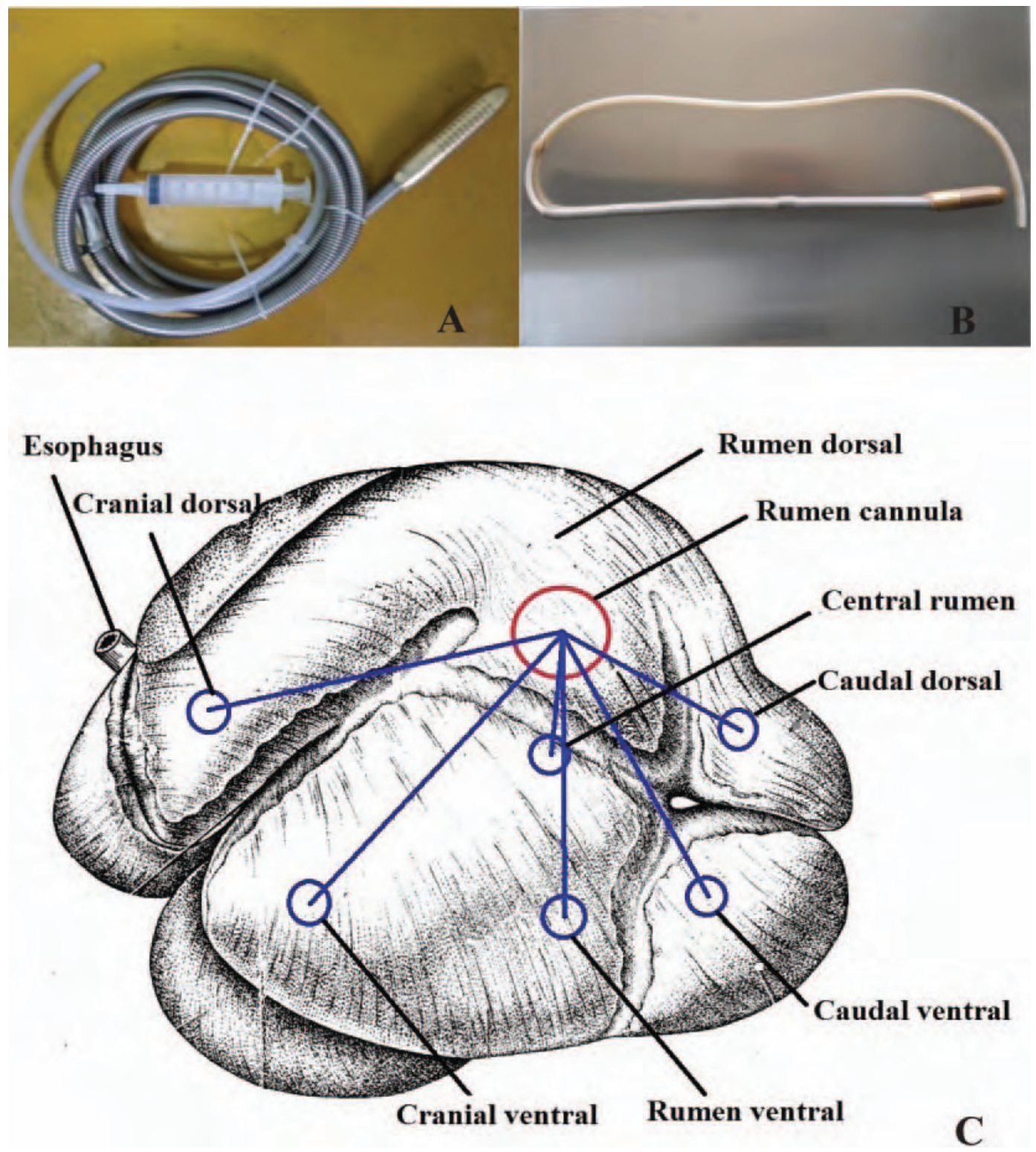

Figure 1. (A) Oral stomach tube with 60-mL syringe, (B) probe used for ruminal fluid collection from rumen cannula, and (C) different sampling sites (rumen picture reproduced from Chen, 2010, with permission of China Agricultural University Press, Beijing). Color version available in the online PDF.

through $\mathrm{RC}$ as described in trial 1 for comparison with that collected via OST.

\section{Chemical Analysis of Samples}

The ruminal fluid taken from the different rumen sites in the rumen (trial 1) or from both techniques (trial 2) was strained through 4 layers of cheesecloth. The $\mathrm{pH}$ of the strained ruminal filtrate was measured immediately using a portable $\mathrm{pH}$ meter (Starter 300; Ohaus Instruments Co. Ltd., Shanghai, China), which was calibrated before each sampling time using stan- dard buffers (pH 4.00 and 6.86). The ruminal fluid collected in wk 3 was only analyzed for $\mathrm{pH}$. For other samples, $5 \mathrm{~mL}$ of filtrate was preserved by adding $1 \mathrm{~mL}$ of $25 \% \mathrm{HPO}_{3}$ for VFA determination, and 2 other 5 - $\mathrm{mL}$ samples of filtrate were stored at $-20^{\circ} \mathrm{C}$ for subsequent analysis of ammonia and ion concentrations.

The rumen filtrate was thawed and centrifuged at $20,000 \times g$ for $10 \mathrm{~min}$. The supernatants were measured for VFA by gas chromatography (GC-8A; Shimadzu Corp., Kyoto, Japan) as described by Hu et al. (2005). One 5-mL sample of each stored ruminal fluid sample was thawed and centrifuged at $3,500 \times g$ for $10 \mathrm{~min}$, 
Table 1. Least squares means of ruminal fermentation parameters for samples collected from the cranial dorsal (CRD), cranial ventral (CRV), central rumen (CR), ventral rumen (RV), caudal dorsal (CAD), and caudal ventral (CAV) cow rumen at approximately 5 to 6 h after morning feeding

\begin{tabular}{|c|c|c|c|c|c|c|c|c|}
\hline Item & CRD & CRV & $\mathrm{CR}$ & $\mathrm{RV}$ & $\mathrm{CAD}$ & CAV & SEM & $P$-value \\
\hline $\mathrm{NH}_{3}-\mathrm{N}, \mathrm{mmol} / \mathrm{L}$ & $4.41^{\mathrm{a}}$ & $3.95^{\mathrm{ab}}$ & $3.70^{\mathrm{b}}$ & $3.51^{\mathrm{b}}$ & $3.57^{\mathrm{b}}$ & $3.51^{\mathrm{b}}$ & 0.18 & $<0.01$ \\
\hline Total VFA, mmol/L & $80.2^{\mathrm{b}}$ & $85.4^{\mathrm{a}}$ & $87.9^{\mathrm{a}}$ & $87.1^{\mathrm{a}}$ & $89.6^{\mathrm{a}}$ & $89.3^{\mathrm{a}}$ & 1.60 & $<0.01$ \\
\hline Propionate, $\mathrm{mmol} / \mathrm{L}$ & $13.4^{\mathrm{b}}$ & $14.3^{\mathrm{a}}$ & $14.8^{\mathrm{a}}$ & $14.6^{\mathrm{a}}$ & $14.9^{\mathrm{a}}$ & $14.9^{\mathrm{a}}$ & 0.31 & $<0.01$ \\
\hline Butyrate, mmol/L & $9.38^{\mathrm{b}}$ & $9.94^{\mathrm{a}}$ & $10.39^{\mathrm{a}}$ & $10.29^{\mathrm{a}}$ & $10.35^{\mathrm{a}}$ & $10.44^{\mathrm{a}}$ & 0.19 & $<0.01$ \\
\hline Acetate:propionate & 4.36 & 4.37 & 4.27 & 4.32 & 4.40 & 4.35 & 0.05 & 0.57 \\
\hline Chloride, $\mathrm{mmol} / \mathrm{L}$ & 16.0 & 16.7 & 16.8 & 15.8 & 16.0 & 15.8 & 0.54 & 0.62 \\
\hline Calcium, mmol/L & 2.42 & 2.52 & 2.45 & 2.38 & 2.36 & 2.61 & 0.11 & 0.58 \\
\hline Phosphorus, mmol/L & 5.72 & 5.69 & 5.82 & 5.81 & 5.75 & 5.97 & 0.16 & 0.86 \\
\hline
\end{tabular}

${ }^{\mathrm{a}-\mathrm{c}}$ Means within a row with different superscripts differ $(P<0.05)$.

and the supernatants were used to determine the ammonia concentrations using the method described by Chaney and Marbach (1962). Another $5 \mathrm{~mL}$ of each stored ruminal fluid sample was thawed and centrifuged at $3,000 \times g$ for $10 \mathrm{~min}$, and the supernatants were analyzed for sodium, potassium, chloride, calcium, and phosphorous concentrations with the color test method using commercial kits (Jiancheng Bioengineering Institute, Nanjing, China).

\section{Statistical Analysis}

The data of the ruminal fluid variables for the samples collected from the different rumen sites or collected via OST and RC were analyzed using the MIXED procedure of SAS (SAS Institute, 2000). The day of collection was a repeated measure (compound symmetry covariance structure). No interactions between site or methods and time of sampling were detected. Thus, the interaction was removed from the model. Differences were considered statistically significant at $P \leq$ 0.05 . The mean separation test was performed using the Tukey multiple range test.

\section{RESULTS}

\section{Differences in Ruminal Fermentation Parameters Among Rumen Sites (Trial 1)}

The fermentation parameters of the samples taken from the different rumen sites are summarized in Table 1. Compared with the ventral rumen or central rumen, the cranial dorsal and cranial ventral rumen had significantly higher $\mathrm{pH}$ values $(P<0.05)$. The caudal ventral and caudal dorsal rumen had moderate $\mathrm{pH}$ values, but the differences were not significant compared with the other rumen sites. The cranial dorsal rumen had the lowest acetate, propionate, and butyrate concentrations compared with the other sites $(P<0.05)$, but no differences were detected among the other sites $(P>0.05)$. The ammonia concentration was not different between the cranial dorsal and cranial ventral sites $(P>0.05)$, but the cranial dorsal rumen had a significantly higher ammonia concentration than the other 4 rumen sites $(P<0.05)$. The cranial dorsal rumen had the highest sodium concentration compared with the other sites. No differences were found in the ratios of acetate to propionate or potassium, chloride, calcium, and phosphorus concentrations among the sites.

\section{Differences in Ruminal Fermentation Parameters Between Sampling Methods (Trial 2)}

The basic information of the 6 rumen-fistulated dairy cows is shown in Table 2. Depending on the measuring results, the OST was inserted to a depth of 180 or $200 \mathrm{~cm}$ for the following comparison of the 2 sampling methods.

When the OST was inserted to a depth of $180 \mathrm{~cm}$, the first batch had a higher $\mathrm{pH}$ value than the other batches $(P<0.05$; Table 3$)$. The first batch had a lower acetate concentration than the fourth batch $(P<$ $0.05)$, but no differences were observed with the other 2 batches $(P>0.05)$. No differences existed among batches in the ammonia, propionate, butyrate, sodium, potassium, chloride, calcium, and phosphorus concentrations $(P>0.05)$. Compared with the ruminal fluid collected via OST, samples collected via $\mathrm{RC}$ had a lower $\mathrm{pH}$ value and sodium concentration, but higher ammonia, acetate, propionate, butyrate, potassium, calcium, and phosphorus concentrations $(P<0.05)$. No difference was observed in the ratio of acetate to propionate or chloride concentrations between the 2 sampling methods $(P>0.05)$. 
Table 2. Identification number, age, BW, and body measurements of the experimental rumen-fistulated cows

\begin{tabular}{|c|c|c|c|c|c|c|c|c|c|}
\hline Item & \multicolumn{6}{|c|}{ Animal identification no. } & Mean & $\mathrm{SD}$ & $\mathrm{CV}, \%$ \\
\hline Age, mo & 42 & 38 & 41 & 41 & 42 & 39 & 41 & 2 & 4.9 \\
\hline \multicolumn{10}{|c|}{ Body measurement. ${ }^{1} \mathrm{~cm}$} \\
\hline HGC & 208 & 201 & 210 & 192 & 194 & 206 & 202 & 7 & 3.5 \\
\hline $\mathrm{BL}$ & 171 & 171 & 167 & 174 & 170 & 171 & 171 & 2 & 1.2 \\
\hline $\mathrm{DCV}$ & 190 & 199 & 197 & 196 & 200 & 202 & 197 & 4 & 2.0 \\
\hline DID & 299 & 217 & 224 & 234 & 222 & 219 & 224 & 6 & 2.5 \\
\hline
\end{tabular}

${ }^{1} \mathrm{HGC}=$ heart girth circumference; $\mathrm{BL}=$ body length; $\mathrm{BH}=$ body height; DIC $=$ distance from the incisors to the cardia of rumen; DIP $=$ distance from the incisors to the front rumen pillar; DCV $=$ distance from the incisors to the center of the rumen; DID $=$ distance from the incisors to the distal point of the caudal-dorsal rumen.

When the OST was inserted to a depth of $200 \mathrm{~cm}$, no differences in $\mathrm{pH}$ value, ammonia, acetate, propionate, butyrate, sodium, potassium, chloride, calcium or phosphorous concentrations were found between the samples collected via OST and RC $(P>0.05$; Table 4$)$.

\section{DISCUSSION}

\section{Sampling Sites Influence Ruminal Fermentation Parameters}

In the current study, the cranial dorsal (atrium) site of the rumen had a higher $\mathrm{pH}$ and sodium concentration and a lower VFA concentration compared with the other rumen sites. These results were consistent with those of previous studies (Bryant and Robinson, 1968; Duffield et al., 2004; Li et al., 2009), even though some differences were found in the other rumen sites. It is known that the atrium (cranial dorsal site) of the rumen is separated from the other rumen sites by the front rumen pillar. During rumination, large amounts of saliva flow into the atrium of the rumen, leading to higher $\mathrm{pH}$ and lower VFA concentrations in the atrium of the rumen compared with other sites (Bryant, 1964). These results indicate that the sampling site may be one main factor contributing to the difference in ruminal fermentation parameters between samples collected via OST and RC.

\section{Insertion Depth of the OST Influences Ruminal Fermentation Parameters}

The distance from incisors to different rumen sites may be related to the breeds and body sizes of cows. According to the measuring results of Geishauser (1993) in 3 German Black Pied cows, the head of OST could reach the ventral sac of the rumen when it was inserted to a depth of $200 \mathrm{~cm}$, which agreed with our results in Holstein dairy cows. Our results also showed that the coefficient of variation of the insertion depth from the

Table 3. Least squares means of ruminal fluid parameters for samples collected via oral stomach tube (different batches, $150 \mathrm{~mL} / \mathrm{batch}$ insertion depth $=180 \mathrm{~cm}$ ) and rumen cannula (ventral rumen)

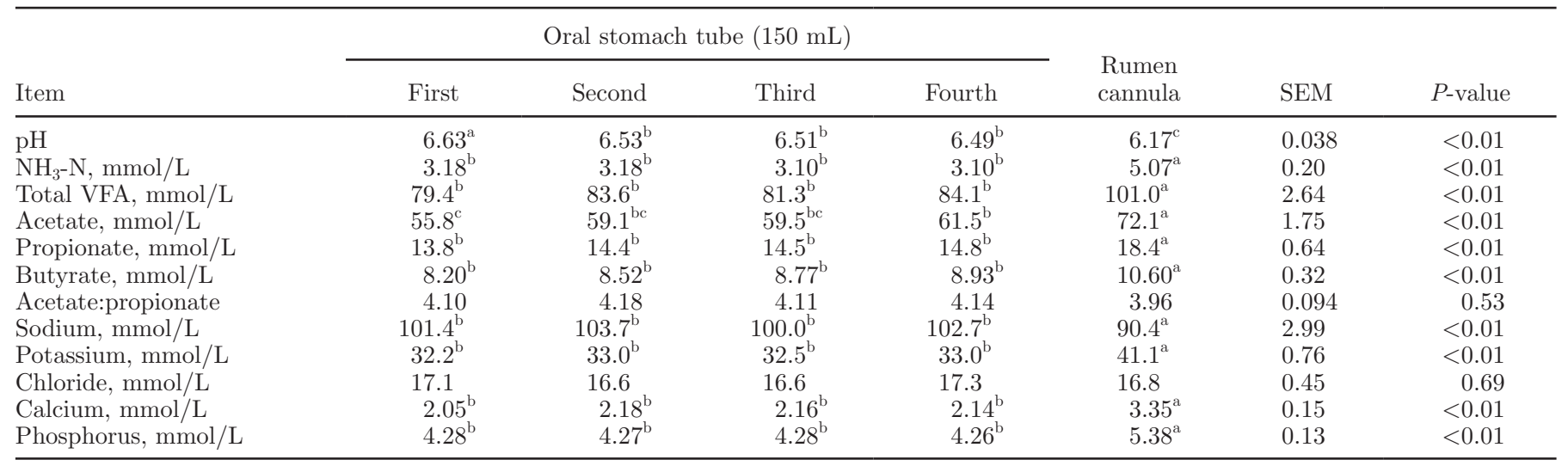

\footnotetext{
${ }^{\mathrm{a}-\mathrm{c}}$ Means within a row with different superscripts differ $(P<0.05)$.
} 
Table 4. Least squares means of ruminal fluid parameter samples collected via oral stomach tube (insertion depth $=200 \mathrm{~cm}$ ) and rumen cannula (ventral rumen)

\begin{tabular}{lcccc}
\hline Item & $\begin{array}{c}\text { Oral stomach } \\
\text { tube }\end{array}$ & $\begin{array}{c}\text { Rumen } \\
\text { cannula }\end{array}$ & SEM & $P$-value \\
\hline $\mathrm{pH}$ & 6.30 & 6.26 & 0.047 & 0.53 \\
$\mathrm{NH}_{3}$-N, mmol/L & 3.10 & 3.17 & 0.20 & 0.80 \\
Total VFA, mmol/L & 89.2 & 94.3 & 1.92 & 0.07 \\
Acetate, mmol/L & 64.1 & 67.3 & 1.38 & 0.12 \\
Propionate, mmol/L & 15.5 & 16.5 & 0.46 & 0.13 \\
Butyrate, mmol/L & 9.60 & 10.10 & 0.26 & 0.19 \\
Acetate:propionate & 4.20 & 4.12 & 0.075 & 0.43 \\
Sodium, mmol/L & 108.7 & 102.1 & 2.72 & 0.14 \\
Potassium, mmol/L & 38.0 & 36.8 & 1.17 & 0.47 \\
Chloride, mmol/L & 16.6 & 16.0 & 0.36 & 0.26 \\
Calcium, mmol/L & 2.48 & 2.46 & 0.11 & 0.91 \\
Phosphorous, mmol/L & 5.46 & 5.59 & 0.16 & 0.57 \\
\hline
\end{tabular}

incisors to the fixed rumen sites was small within the observed $\mathrm{BW}$ range $(\mathrm{BW}=604 \pm 36 \mathrm{~kg}$, mean $\pm \mathrm{SD}$; Table 2). However, for other breeds or body sizes of cows, the insertion depth of OST from incisors to different rumen sites should be confirmed in further studies.

In this study, when the OST was inserted to a depth of $180 \mathrm{~cm}$, the OST head was located in the cranial dorsal (atrium) site of the rumen. The ruminal fluid collected via OST had a higher $\mathrm{pH}$ and higher sodium concentration, but lower VFA, potassium, calcium, and phosphorus concentrations than those of fluid collected via RC (Table 3). Raun and Burroughs (1962) and Wagner (1984) obtained similar results in $\mathrm{pH}$ and VFA concentrations and attributed them to the saliva contamination. From Table 3, it can be seen that ruminal $\mathrm{pH}$ for the OST samples was approximately 0.32 to 0.46 higher than samples collected from $\mathrm{RC}$ when the OST was inserted to a depth of $180 \mathrm{~cm}$. However, the results of trial 1 (Table 1) showed that the ruminal $\mathrm{pH}$ in the cranial dorsal area was only approximately 0.17 higher than that in the rumen ventral area. Therefore, in addition to the sampling site, some other factors may also contribute to the higher ruminal $\mathrm{pH}$ for samples collected via OST. The ruminal $\mathrm{pH}$ of the first batch was approximately 0.10 to 0.14 higher than the other batches, indicating that saliva entering the head of the OST during insertion may be another reason for the contamination of the ruminal fluid. Duffield et al. (2004) observed similar results and recommended discarding the first $200 \mathrm{~mL}$ of ruminal fluid. Also, Dirksen (1975) suggested that the flow of saliva induced by probing, passing from the reticulum to the atrium of the rumen, can also add to the severity of saliva contamination. Therefore, shortening the sampling time could also help reduce saliva contamination.

When the OST was inserted to a depth of $200 \mathrm{~cm}$, the OST head could penetrate the fiber mat, pass through the front rumen pillar, and reach central rumen, even though the OST head may be located more in the rumen dorsal or ventral area. No differences were observed in $\mathrm{pH}$ and VFA, ammonia, and ion concentrations between samples collected via the 2 sampling methods by discarding the first $150 \mathrm{~mL}$ of ruminal fluid collected (Table 4). These data agree with Geishauser (1993) and Geishauser and Gitzel (1996), who considered that saliva contamination can be avoided effectively. In this study, we did not compare the difference between OST samples collected under 2 different insertion depths directly. However, the comparison of the 2 sampling methods indicated that the insertion depth of the OST may affect ruminal fermentation parameters. To reduce saliva contamination and increase the representativeness of ruminal fluid collected in dairy cows, the OST should be inserted into the central rumen for sampling, the first $150 \mathrm{~mL}$ of ruminal fluid should be discarded, and the sampling time should be shortened.

Today, various types of OST are used in ruminal fluid collection. Some kinds of OST use rubber (Raun and Burroughs, 1962; Hofírek and Haas, 2001), plastic (Enemark et al., 2004), or poly tubes (Lodge-Ivey et al., 2009) as a suction or nested tube. However, these OST are too soft to penetrate the fiber mat, and the ruminal fluid can only be collected from the cranial dorsal (atrium) site of the rumen. However, the Dirksen and Smith (1987) and Geishauser (1993) probes were modified OST. In these probes, the outside of the soft suction tube was nested with a stainless spiral spring, which guaranteed that it could penetrate the fiber mat, pass through the front rumen pillar, and reach the central rumen for sampling. Therefore, to guarantee the reliability and comparability of different studies, it is important to provide a detailed description of the types of OST used and how deeply they are inserted when using them for ruminal fluid collection. 


\section{CONCLUSIONS}

The results of the present study demonstrate that the insertion depth of the OST may affect the fermentation parameters of the ruminal fluid collected in dairy cows. The sampling site may be one of the main reasons for the difference observation of ruminal fermentation parameters for the samples collected under the 2 different OST insertion depths. Inserting the OST into the central rumen for fluid sampling could reduce saliva contamination and increase the representativeness of the ruminal fluid.

\section{ACKNOWLEDGMENTS}

We thank the staff of the Institute of Dairy Science (Hangzhou, China) and Shucong Li (University of Manitoba, Winnipeg, MB, Canada) for their technical assistance. We also acknowledge the personnel of the Hangzhou Zhengxing dairy farm (Hangzhou, China) for their assistance with animal care.

\section{REFERENCES}

Bryant, A. M. 1964. Variations in the $\mathrm{pH}$ and volatile fatty acid concentration within the bovine reticulo-rumen. N. Z. J. Agric. Res. 7:694-706.

Bryant, M. P., and I. M. Robinson. 1968. Effects of diet, time after feeding, and position sampled on numbers of viable bacteria in the bovine rumen. J. Dairy Sci. 51:1950-1955.

Chaney, A. L., and E. P. Marbach. 1962. Modified reagents for determination of urea and ammonia. Clin. Chem. 8:130-132.

Chen, Y. X. 2010. Anatomy of the Domestic Animals and Fowl. 3rd rev. ed. China Agricultural University Press, Beijing, China. (In Chinese.)

Dirksen, G. U. 1975. Eine lenkbare Sonde zur gezielten Entnahme von Pansensaft beim Rind (Rumen sampling in cattle using a maneuverable probe). Tierärztl. Umsch. 30:367-370.

Dirksen, G. U., and M. C. Smith. 1987. Acquisition and analysis of bovine rumen fluid. Bovine Pract. 22:108-116.
Duffield, T., J. C. Plaizier, A. Fairfield, R. Bagg, G. Vessie, P. Dick, J. Wilson, J. Aramini, and B. McBride. 2004. Comparison of techniques for measurement of rumen $\mathrm{pH}$ in lactating dairy cows. J. Dairy Sci. 87:59-66.

Einarson, M. S., J. C. Plaizier, and K. M. Wittenberg. 2004. Effects of barley silage chop length on productivity and rumen conditions of lactating dairy cows fed a total mixed ration. J. Dairy Sci. $87: 2987-2996$.

Enemark, J. M., R. J. Jorgensen, and N. B. Kristensen. 2004. An evaluation of parameters for the detection of subclinical rumen acidosis in dairy herds. Vet. Res. Commun. 28:687-709.

Geishauser, T. 1993. An instrument for the collection and transfer of ruminal fluid and for the administration of water soluble drugs in adult cattle. Bovine Pract. 27:38-42.

Geishauser, T., and A. Gitzel. 1996. A comparison of rumen fluid sampled by oro-ruminal probe versus rumen fistula. Small Rumin. Res. 21:63-69.

Hofírek, B., and D. Haas. 2001. Comparative studies of ruminal fluid collected by oral tube or by puncture of the caudoventral ruminal sac. Acta Vet. (Brno) 70:27-33.

Hook, S. E., K. S. Northwood, A. D. Wright, and B. W. McBride. 2009. Long-term monensin supplementation does not significantly affect the quantity or diversity of methanogens in the rumen of the lactating dairy cow. Appl. Environ. Microbiol. 75:374-380.

Hu, W. L., J. X. Liu, J. A. Ye, Y. M. Wu, and Y. Q. Guo. 2005. Effect of tea saponin on rumen fermentation in vitro. Anim. Feed Sci. Technol. 120:333-339.

Li, M., G. B. Penner, E. Hernandez-Sanabria, M. Oba, and L. L. Guan. 2009. Effects of sampling location and time, and host animal on assessment of bacterial diversity and fermentation parameters in the bovine rumen. J. Appl. Microbiol. 107:1924-1934.

Lodge-Ivey, S. L., J. Browne-Silva, and M. B. Horvath. 2009. Technical note: Bacterial diversity and fermentation end products in rumen fluid samples collected via oral lavage or rumen cannula. J. Anim. Sci. 87:2333-2337.

Nocek, J. E. 1997. Bovine acidosis: Implications on laminitis. J. Dairy Sci. 80:1005-1028.

Nordlund, K. V., and E. F. Garrett. 1994. Rumenocentesis: A technique for the diagnosis of subacute rumen acidosis in dairy herds. The Bovine Practitioner 28:109-112.

Raun, N. S., and W. Burroughs. 1962. Suction strainer technique in obtaining rumen fluid samples from intact lambs. J. Anim. Sci. 21:454-457.

SAS Institute. 2000. SAS User's Guide: Statistics. Version 8.01. SAS Inst. Inc., Cary, NC.

Wagner, D. 1984. Comparative testing of four probes for rumen sampling in adult cattle under consideration of saliva influx to the sample. Diss. Ludwig-Maximilians-Universität, Munich, Germany. 